\title{
e-Phaïstos
}

e-Phaïstos

Revue d'histoire des techniques / Journal of the history

of technology

II-1 | 2013

Savoirs et sciences sur les machines

\section{Mécaniser la perspective : les instruments entre pratique et spéculation}

Mechanising Perspective: Instruments between Practice and Speculation

\section{Pascal Dubourg-Glatigny}

\section{OpenEdition}

\section{Journals}

Édition électronique

URL : http://journals.openedition.org/ephaistos/1381

DOI : 10.4000/ephaistos. 1381

ISSN : 2552-0741

\section{Éditeur}

IHMC - Institut d'histoire moderne et contemporaine (UMR 8066)

\section{Édition imprimée}

Date de publication : 1 juin 2013

Pagination : 23-33

ISSN : 2262-7340

\section{Référence électronique}

Pascal Dubourg-Glatigny, « Mécaniser la perspective : les instruments entre pratique et spéculation », e-Phaïstos [En ligne], II-1 | 2013, mis en ligne le 15 décembre 2019, consulté le 18 juin 2020. URL : http://journals.openedition.org/ephaistos/1381 ; DOI : https://doi.org/10.4000/ephaistos.1381 


\title{
Mécaniser la perspective : les instruments entre pratique et spéculation
}

\author{
Pascal Dubourg-Glatigny \\ CNRS, Centre Alexandre Koyré
}

La question de la mécanisation des arts à la Renaissance est liée à la définition des conditions d'exercice du dessin. L'apprentissage est fondé sur l'imitation des modèles du passé, parmi lesquels il convient de choisir non seulement la manière de représenter la plus excellente mais aussi la plus adaptée à une situation donnée. La pratique se traduit par la mise en œuvre de procédés usuels, ponctuellement et partiellement généralisés sous la forme de règles, auxquels les moyens mécaniques peuvent apporter un complément plus fiable, plus efficace et plus rapide. À la rigueur de la main, se substitue la certitude des mathématiques. En effet, les mathématiques mixtes, auxquelles la perspective appartient, puisent, dans la connaissance des quantités nombrables et mesurables, leurs ressources pour éclairer, démontrer et transformer la matière. Ce phénomène contribue à estomper l'ancienne frontière qui éloignait connaissance et production et réunit désormais dans une communauté de destin toutes les disciplines qui, à travers la mesure du sensible, conduisent à la formulation graphique. Elles sont nombreuses; on trouve cependant à leur tête la cosmographie et l'architecture, deux disciplines dont le périmètre a été défini par les traités des Anciens. En lien avec les mathématiques pures (géométrie et arithmétique), elles enseignent à construire une carte ou un édifice, deux objets qui, à des échelles différentes, sont autant de moyens d'étendre la connaissance de l'espace. $\mathrm{Si}$ « la perspective » est une discipline relevant elle aussi du domaine de la connaissance du monde, à la rencontre entre géométrie, astronomie et optique, « le dessin en perspective » constitue pour sa part une opération matérielle. Dans un contexte culturel où la perception du réel passe à travers le prisme d'une vision idéaliste de la nature, la distinction entre perspective spéculative et perspective artificielle ou l'assimilation de l'une dans l'autre fait l'objet d'une redéfinition permanente. Celle-ci dépend non seulement du contexte, mais aussi de la formation des acteurs et de la finalité de leurs activités. Il n'est ainsi pas possible d'en décrire l'évolution de manière linéaire et purement chronologique.

Ce débat intellectuel s'accompagne d'une réalité plus concrète. La mise en œuvre de savoirs spéculatifs, ou plus exactement le mariage des intérêts des savants avec ceux des praticiens rencontre, depuis la fin du XVe siècle, une reconnaissance croissante de la société. La diffusion des connaissances due à l'imprimerie et au développement des universités joue, dans ce processus, une place significative. Et cet intérêt théorique se traduit logiquement par une attente du public pour une application plus large et plus fréquente de ces savoirs et des savoir-faire qui en découlent. Dans ces conditions, la mécanisation des opérations constitue un enjeu primordial de leur développement. Qu'il s'agisse de l'art de la guerre, de l'art de bâtir ou de celui de mesurer la terre, le développement des machines s'imposa comme un outil garantissant la validité géométrique à l'occasion d'un usage démultiplié par un personnel nombreux aux qualifications variables. L'apparition, au cours du XVIe siècle, du métier de perspecteur - un peintre spécialisé dans le dessin de la perspective -, participe de ce mouvement. Cette 
figure professionnelle, encore mal identifiée par l'histoire de l'art, a sans doute participé au débat sur l'introduction et l'usage des machines de perspective dans les ateliers, un débat malheureusement guère documenté, dont il convient de reconstruire les termes à travers ce que les traités publiés nous ont légué.

Mais qu'est-ce qu'une machine pour les opérateurs de la Renaissance ? Quelle fonction lui attribue-t-on, quelle est sa finalité ? La machine constitue-t-elle un intermédiaire entre la théorie et la pratique ou possède-t-elle un autre statut ? Voici les questions que nous aimerions poser à travers l'exemple du développement des machines à dessiner la perspective à la Renaissance.

\section{Macchina - ordigno - strumento}

Le vocabulaire de la Renaissance italienne connaît trois termes principaux pour désigner les appareils techniques de production. Le mot in/strumento est celui qui possède le plus vaste champ sémantique, le vocable macchina est, par contraste, d'un usage plus restreint. On trouve enfin le mot ordigno, substantif plus récent, le seul des trois s'avérant ne pas être le calque d'un mot latin ou grec. Afin de saisir quelle valeur on attribuait à ces mots, il convient, comme dans bien des cas, de se replier sur le dictionnaire de l'Académie de la Crusca, dont la première édition en 1612 illustre l'usage lexical affirmé au cours du XVI' siècle'$^{1}$.

Un témoin un peu plus ancien était cependant utile: nous avons parallèlement eu recours aux manuscrits de Léonard, qui nous fournissent ainsi un point d'appui pour approcher l'usage du vocabulaire au $\mathrm{XV}^{\mathrm{e}}$ siècle ${ }^{2}$. Pour Léonard, macchina se réfère principalement aux machines de guerre et de construction, un équipement technique de grande dimension. Cela s'insère dans la tradition de Vitruve, qui regroupait dans le livre $\mathrm{X}$ du De Architectura les machines opérant sur la mécanique : celles destinées à la traction et à l'élévation, à l'hydraulique et à la poliorcétique. Dans le glossaire contenu dans le codex Trivultien, il ne manque cependant pas de mentionner «macchina: edifitio »3. La notion de "machine» ne s'applique donc pas seulement à des outils de production mais à un organisme constitué selon les règles de l'art, en particulier à l'architecture. L'usage que Léonard fait du terme strumente/o est beaucoup plus large et se réfère à tout opérateur de changement ou moyen d'interprétation qu'il soit mécanique, physique ou intellectuel.

Les termes macchina/strumento sont cependant parfois interchangeables. Dans la description de «l 'Architronite », machine de guerre ainsi nommée en l'honneur d'Archimède, propulsant les projectiles au moyen de la pression de la vapeur d'eau, il amorce ainsi son discours : "L'architronite est une machine en cuivre fin » mais quelques mots plus loin, fournit le détail de la «troisième partie de cet instrument $\gg 4$. Dans une note en marge d'un dessin anatomique datant de 1513, Léonard désigne le corps humain par le terme de macchina et la dissection par celui de strumento 5 . L'instrument est alors le procédé intellectuel répondant à certaines règles et se traduisant par une opération matérielle. La macchina est un ensemble, généralement d'une certaine ampleur, de parties coordonnées pour parvenir à un résultat. Elle constitue un sous-ensemble de la catégorie des instruments, beaucoup plus large. En effet, si la machine ne renvoie qu'à des constructions de l'art ou de la nature, l'instrument peut être soit matériel, soit intellectuel.

Le terme macchina renvoie directement à l'action, macchinazione, qui recouvre, selon la Crusca, trois significations : le recours à la ruse et à l'artifice, l'instrument ou machine de guerre et enfin, le sens artistique de machine qui désigne les grands et nobles édifices de l'architecture ${ }^{6}$. On pense alors à la machina infernale qui, selon l'Arioste, enseigna à toutes les nations un nouvel art cruel de la guerre ${ }^{7}$ ainsi qu'à la «superbe machine de la coupole de Sainte-Marie de la Fleur » dont Vasari fait l'éloge ${ }^{8}$. Par extension, le mouvement cinétique est inclus dans les machines et désigne alors le monde, l'uni- 
vers comme des machines. C'est la machina mundi platonicienne, œuvre parfaite du créateur, dont les théoriciens de l'héliocentrisme s'emparèrent avec véhémence. Cet usage se serait répandu depuis la traduction, semble-t-il très libre, du Timée par saint Augustin9.

Entre la macchina et le strumento, on trouve en italien le terme ordigno, dérivé du latin ordinare qui renvoie à la mise en ordre, à la disposition, à l'agencement. L'ordigno est, selon le dictionnaire de la Crusca, une " chose qui relève de l'art, le nom générique d'un instrument composé avec art, en vue de différentes opérations ${ }^{10}$. On y trouve plusieurs objets qualifiés d'ordigno : le travail à ferrer, la cage des oiseaux, la muselière des animaux... Ils sont assez rares et ce sont, comme on le voit, plutôt des objets techniques que des machines. L'ordigno est cependant souvent considéré comme l'équivalent d'un in/strumento. Il est considéré comme un mot plus populaire ${ }^{11}$, d'origine lombarde, si l'on suit le lexicologue Francesco Alunno (1562) ${ }^{12}$.

L'ordigno/strumento est ce « avec quoi ou par le moyen duquel nous opérons »13. Et de donner parmi les exemples dans lesquels les instruments techniques côtoient les instruments de musique une série d'instruments de géométrie et d'astronomie, comme la règle, le quadrant ou le compas. Mais l'adjectif strumentale est aussi un «terme philosophique » qui nous conduit vers des significations plus larges. Ainsi « la mesure », notion opératoire et non machine, est décrite comme la « distinction déterminée de la quantité et instrument par lequel on établit la différence »14. Comme on l'a vu chez Léonard et comme on le constate chez les auteurs de médecine, le corps humain est lui aussi composé de quantité d'instruments: ainsi l'œil est l'instrument de la vue ${ }^{15}$.

\section{Les premières machines de perspective}

Les instruments de perspective sont principalement connus à travers l'intersecteur décrit par Alberti dans le De Pictura (1435) et les nombreux instruments qui en dérivent. Mais s'agit-il vraiment d'une machine? C'est « un voile de fils très fins au tissage lâche [...] divisé par des fils plus gros en autant de carrés alignés que [l'on] voudra et tendu sur un cadre ${ }^{16}$. Ce panneau est placé entre l'observateur et l'objet représenté. Il procure un quadrillage sur lequel on déduit les coordonnées du dessin. Alberti ne présente pas l'intersecteur comme un instrument et encore moins comme une machine; c'est une chose (rebus) pour laquelle il ne trouve pas de qualificatif approprié. Disons donc qu'il s'agit d'un dispositif technique destiné à matérialiser le processus de la vision naturelle au moment de l'exercice du dessin. Dans les faits, Alberti n'explique pas comment on procède au report des données visuelles sur le papier, laissant de côté les instructions de mise en œuvre, la procédure. L'intersecteur permet de peindre "facilement et correctement »: le texte se limite à des préceptes sans fournir une description véritablement technique. Puisque le dispositif répond à une exigence de rationalisation du dessin, Alberti qui, outre les préceptes, énonce beaucoup de principes, s'emporte contre ceux qui « déclarent tout à fait inutile que le peintre se familiarise avec ces choses ${ }^{17}$. L'intersecteur répond donc avant tout à une exigence théorique fondée sur la rationalisation géométrique et non à une contrainte de production passant par la mécanisation des opérations ${ }^{18}$. L'instrument incarne une théorie.

Les fondements de ce dispositif ont été rendus célèbres par les gravures de Dürer montrant l'emploi de son «portillon» (Thürlein), décliné de nombreuses manières : tantôt en portrait, tantôt avec un luth, tantôt avec une femme nue couchée. À travers la diversité des sujets représentables, on passe clairement du principe à la mise en œuvre. Dürer expose explicitement un instrument (das Zeug) : son caractère productif est cependant aujourd'hui regardé comme ambigu, comme on peut le constater dans les dernières traductions publiées en 1995. En effet, les deux versions françaises du traité hésitent sur ce terme. Van Peene traduit Zeug 
par « appareil », adhérant littéralement au caractère technique alors que Peiffer choisit "dispositif », laissant planer quelque doute sur la fonctionnalité du portillon. Plus loin, alors que Dürer n'éprouve pas le besoin de qualifier son instrument (nun gebrauche dies also), Peiffer introduit un substantif («utilise ce dispositif comme suit»), abandonnant l'indétermination du pronom démonstratif alors que Van Peene reste plus proche de la phrase de Dürer. Plus curieusement, le second traducteur emploie cependant "dispositif » pour traduire la Meinung conclusive qui, en sa qualité d'opération purement intellectuelle, est rendu par Peiffer comme « conception »19.

Contrairement à celui d'Alberti, l'instrument de Dürer s'inscrit totalement dans le contexte des ateliers : on y trouve non seulement le descriptif de sa construction mais aussi les modalités de son emploi. Cependant les historiens des mathématiques regardent les deux plutôt comme des instruments conceptuels, des dispositifs démonstratifs, alors que les historiens de l'art pensent spontanément à la production de la peinture. Mais la distinction entre les deux instruments, l'un théorique, l'autre productif, reste dans l'ombre de leur similitude.

\section{Mesurer et représenter l'espace, questions d'échelles}

Les instruments appartenant à la grande famille des intersecteurs ou des portillons possèdent toutefois quelques caractéristiques communes. Ils sont fondés sur l'observation d'un objet à une distance déterminée et permettent de visualiser le rapport mesurable entre cet objet et l'observateur. Dans l'espace réel, cette opération se produit dans la pyramide formée par le champ de vision réduit à l'angle de vue. La traduction dans l'espace représenté, la perspective, consiste alors à transposer les rapports de proportion constatés dans la pyramide, espace en trois dimensions, en un triangle, espace en deux dimensions. Sur un plan pratique, il s'agit donc de relever l'impact des formes sur la coupe de cette pyramide, simulacre du tableau. On le comprend, le rapport à l'espace mesurable - et non plus seulement intuitivement perceptible -, fonde la théorie de la perspective. Il n'est pas difficile de penser comment les exigences métriques qui présidaient à la connaissance de l'éloignement des lieux qu'ils soient célestes, maritimes ou terrestres -, ont pu s'étendre aux objets qui composent accidentellement ces lieux. L'outil intellectuel servant à l'astronome pour déterminer la distance des étoiles pouvait ainsi être employé, à une échelle plus réduite, par l'arpenteur calculant l'étendue des terrains agricoles ou des forêts et, à une échelle encore inférieure, au peintre déterminant le raccourci des objets situés dans l'espace intermédiaire. C'est cette articulation d'échelles de représentations d'un même ensemble plus vaste qui a été théorisée à la Renaissance, par exemple dans les pratiques cartographiques introduisant une distinction entre cosmographie et chorographie ou dans celles de composition des tableaux articulant le paysage ou l'architecture avec les groupes de figures. Dans la pratique, la généralisation de l'impératif métrique à cette époque a naturellement démultiplié les activités et les opérateurs qui y étaient soumis, exerçant alors une pression pour une mécanisation des procédures assurant une plus grande rapidité et une plus grande fiabilité.

Dans l'Optique, Euclide avait enseigné comment appliquer la théorie des triangles semblables pour la mesure des distances inaccessibles: en dehors des miroirs, il n'envisageait cependant pas le recours à un quelconque instrument. On doit à son premier traducteur moderne, Egnatio Danti (1573), d'avoir précisé le passage de ces pratiques à la $m a-$ chinatio dans les commentaires qu'il prend soin d'ajouter à cet ouvrage de l'Antiquité. En effet, si dans les théorèmes 18 à 21, Euclide enseigne la proportionnalité d'aires de triangles de hauteur égale, c'est Danti qui, en annotation, indique le recours à une échelle altimétrique ou à « tout autre instrument » pour mesurer ${ }^{20}$. 


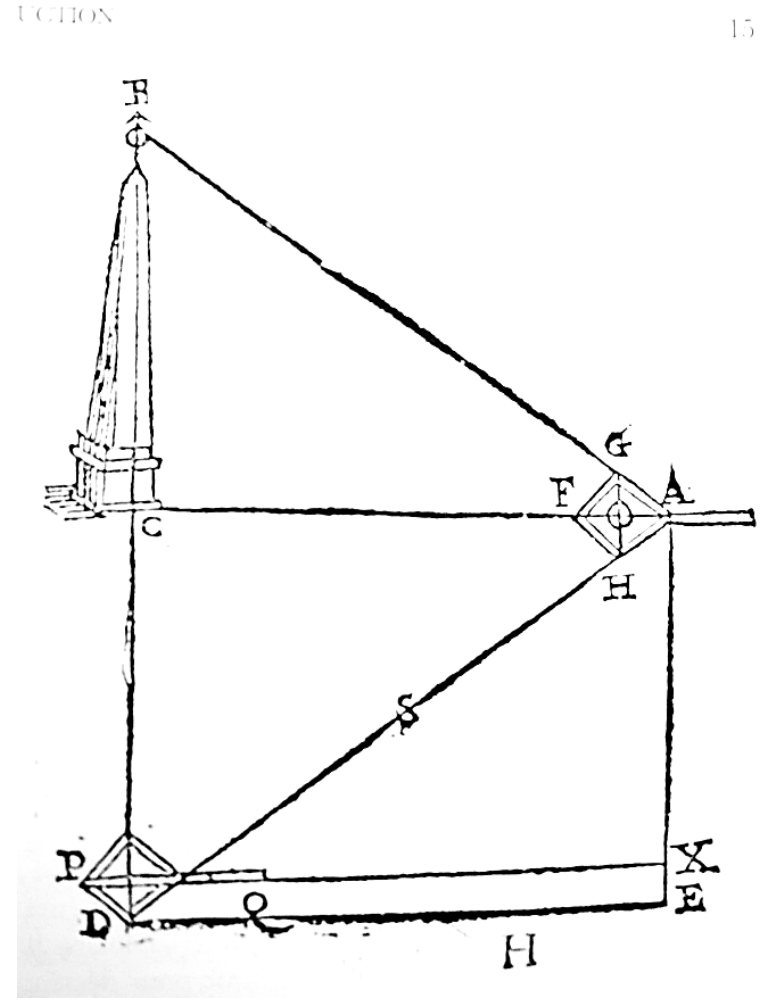

Fig. 1: La mesure des distances dans Latino Orsini, Egnatio Danti, Trattato del radio latino, Rome, 1586 [1583], p. 10

Plus détaillée est encore l'instrumentation présentée par Danti pour appuyer le 1er théorème de la Catoptrique dont voici la définition euclidienne: « les rayons visuels se reflètent en des angles égaux, tant dans les miroirs plats, que dans ceux qui sont convexes et ceux qui sont concaves ${ }^{21}$. En complément, Danti suggère une "expérience », proposant à l'opérateur de «faire un instrument »: son traité est le premier de la Renaissance à fournir une description détaillée des principes de la camera obscu-
D I I V C.I. I D E.

fono riportate fotto fopra. Hor fe torremo, che l'imagini tornino per il uer fo loro, metteremo uno $\beta$ pecthio fotto il bugio dentro alla fineftra comenella prefente figurafi uede lo pecebio.c D. aumili bugio, nel quale percuotédo il raggio. A c. che efee dalla punta della pi ramide fi riflette ad angolipari,oripor ta detta punta nel muro al punto. $\mathrm{s}$. coff i raggi, che efco no da' punti. c. or

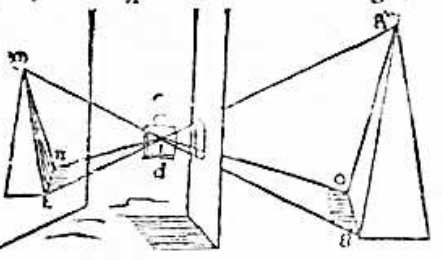

.o. della bafa riffettendofi ad angoliparifi improntono ne' pumi del muro. $\mathrm{N}$ L. La onde la piramide, L N M. cagionata da' raggi, che nello pecclio perctotendo fi riflettono, \& non caminono rettamente, uien figurata peril uerfo fuo come fta quella, che la cagiona. E ben uero, che quefle imagini, che fono cagionate da raggirefleffi non $/ i$ uedono cofi fcolpite, ne dift siui colori, come fanno quelle, che da rag gi retri fono caufate. Terche iragyi infuali refieffs, fono piu debili, cbe non fono iretti, Et di qui nafce, che noi non ci ricordiamo dell'imagine del nof tro nifo, che uediamo nello peccbio, come ci ricordiamo delle imagini degl'altri, perclsc la nofira la uediamo co' raggi reficff, che non imprimono nella memoria, come finno i raggireti, co' quali tuediamo il uifo degl'altri. Ft quefto intefe S. Iacopo nel pri mo cap. della fua Epiftola quando diffe. Si quis auditor eft uerbi, \& non factor: bic comparabitur uiro confideranti unltsm natiuitatis fux in peculo; confiderasit cnim fe, of abiit, \& ftatim oblitus cst, qualisfuerit. Reftabor folo à dire, che quanto il muro farà piu uicino ò lontano dal bugio tanto farà maggiore, ò minorc la cofa rap prefentata, percle fe il miro foffe tanto lontano dal bugio, quanto di fwori clontana la piramide, quclla rapprefentata faria eguale alla uera, \& ciò fi proua per la $\mathrm{s}$. del primo, perche gl'angoli opposti nelle punte, che percuotono nello fpecebio fono cguali, bor fe i due la ti di un triangolo fono eguali à due lati dell'altro trianzolo, anco la ba fa, che cl altezza d'una piramide farà eguale all'altra bafa, che laltezza dell altra piramide, per la quarta del primo; Da queft a fie rienza, if fuopre anco la cagione, ond c proceda, che iraggi del Sole, che entrono per ibugidelle fineftre, che banno pin faccic, o piu an goli, glinediamo in terra di forma rotonda. Perche talc ch la natura delle cofe, che di raggi fono riport.te fecondo la forma loro come $\mathrm{L} 2$ apparc

Fig. 2 : La Camera oscura d'Egnatio Danti dans La prospettiva di Euclide, Florence, 1573, p. 83 .

$r a$. Elle possède ici le statut d'un dispositif de démonstration théorique et non d'un instrument destiné à la production picturale ${ }^{22}$. La machine n'est ici pas en jeu puisque la camera obscura ne requiert pas une construction spécifique, produit d'une compétence dans un art mécanique : il suffit de se 
placer dans la pièce d'une maison et, après avoir réalisé un petit orifice dans le mur extérieur, d'observer le mouvement sur la place ou dans la rue à travers l'image produite sur le mur qui fait face à l'orifice. Ces « images » peintes évoluent, nous dit Danti, dans la dipintura ${ }^{23}$. Il n'est pas ici question de représentation dessinée, notamment car l'intérêt du dispositif est de montrer une image en mouvement alors que la peinture ne peut produire qu'une image fixe. Il s'agit d'isoler un phénomène naturel pour en saisir, à travers l'expérience, les conséquences conceptuelles. Plus tôt, on le sait, Léonard avait déjà exposé visuellement les principes de la camera oscura mais comme il l'a été montré pour les pages qu'il consacre aux miroirs, il n'en retira pas d'usage mécanisé à destination de la peinture ${ }^{24}$.

Ailleurs dans l'ouvrage et notamment dans la préface à l'Optique d'Euclide, Danti traite des questions de mécanisation, mais il s'attache surtout à préciser que les «instruments produits par l'art nous montrent que les rayons sont portés par une ligne droite ${ }^{25}$.

Ces instruments physiques, fatti dall'arte, sont des machines théoriques qu'il convient de distinguer toutefois des instruments intellectuels : dans le cas présent, ils mettent matériellement en évidence les caractères sensibles des phénomènes naturels.

\section{L'instrumentation et la démonstration pers- pective}

Dans son édition de 1586 consacrée aux règles de perspective de Vignole, jusqu'alors restées manuscrites, Danti présente un catalogue composé de huit instruments inventés ou employés par les artistes $^{26}$. Une première série se fonde sur le portillon de Dürer : ceux-ci visent à montrer " dans les faits » (in atto) les principes de la perspective dessinée ce qui «permet aux artistes de découvrir plus facilement cette merveilleuse invention ${ }^{27}$.

Par la similitude entre l'image vue dans le cadre et l'image dessinée, «l'œuvre doit paraître vraie et tromper la vue des spectateurs ». Les instruments

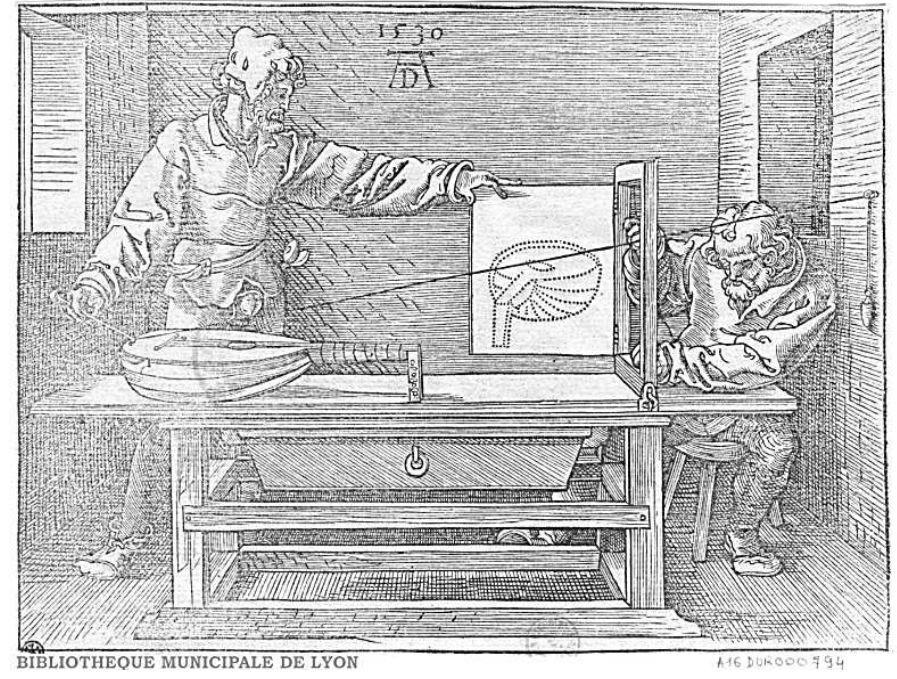

Fig. 3 : Le portillon de Dürer, 1530 [1525], Bibliothèque municipale de Lyon.

procurent les coordonnées des objets soit par un système de fils, soit par un couple de réglettes amovibles améliorant la précision par rapport au système de quadrillage employé par Dürer. En effet, celui-ci permet d'obtenir un réseau de repères, plus ou moins dense selon le quadrillage, mais qu'il est toujours nécessaire de compléter par la «pratique ", c'est-à-dire à main levée. Les fils amovibles ou les réglettes permettent en revanche de suivre plus exactement les contours des figures et de placer des points de repère à un espacement variable : plus serré lorsque les courbes deviennent plus complexes, plus lâches lorsque la ligne tend à être droite. L'avantage n'est pas seulement technologique, il est également conceptuel: la traduction matérielle de l'idée, le passage entre le dessin interne et le dessin externe, pour reprendre le vocabulaire de Zuccaro, est plus assuré. L'instrument permet de parvenir à un résultat plus fidèle que l'usage de la règle géométrique, dont la mise en œuvre dépend des conditions physiques de l'opérateur. Cependant, l'emploi de quelques instruments, comme 
certains portillons à filet qui nécessitent une manipulation qualifiée de «pratique », réintroduit une incertitude. Lorsque la main vacille, la règle, aussi mathématique qu'elle soit, n'y peut rien ! Danti le reconnaît lui-même bien volontiers : «Il est si difficile que la main puisse obéir à ce que l'esprit lui suggère !» ${ }^{28}$.

L'instrument le plus célèbre que présente Danti est le distanciomètre de Baldassare Lanci, dont un exemplaire est conservé au Musée d'histoire des sciences de Florence ${ }^{29}$. Il est composé de plusieurs accessoires combinables pour parvenir à différentes opérations comme le relevé cartographique, la mesure des hauteurs à distance ou même, comme il a été avancé, l'établissement du plan d'une forteresse d'après son relevé perspectif $3^{\circ}$. Un viseur entraîne sur un axe central un stylet fixé en parallèle. Celuici désigne, sur une feuille de papier collée à un semi-tambour perpendiculaire, les points de repère correspondants aux angles observés. Cet instrument possède l'avantage de la mobilité : on peut orienter le viseur latéralement et horizontalement et l'établissement des points servant à la représentation s'effectue directement, sans devoir relever manuellement les coordonnées, comme l'obligent les différents portillons. Selon Danti, si la perspective reste exacte lorsque le papier est fixé sur le tambour, elle devient évidemment fausse lorsque le relevé est posé à plat. Il a été montré que cet instrument mettait en œuvre le principe de la projection isogone orthogonale équatoriale exposée par Mercator $^{31}$. En effet, le tambour permettait de prendre en compte la courbure de la terre que la perspective figurée, contrainte par son exigence de représentation plane, ignore ou considère comme marginale à cette échelle de perception.

Le distanciomètre de Lanci conservé à Florence n'est pas seulement un objet pratique. Il est même assez improbable que cet exemplaire ait été employé sur le terrain. Il semble qu'il fît partie des collections de la salle des cartes de géographie du Palazzo Vecchio bien que Danti, qui y travailla longtemps, ne le mentionne pas. Fabriqué en laiton, reflétant des couleurs dorées brillantes, il est composé d'une tablette sur laquelle une scène figurant l'usage qu'on peut en faire a été gravée avec soin. C'est donc à la fois un instrument et la représenta-

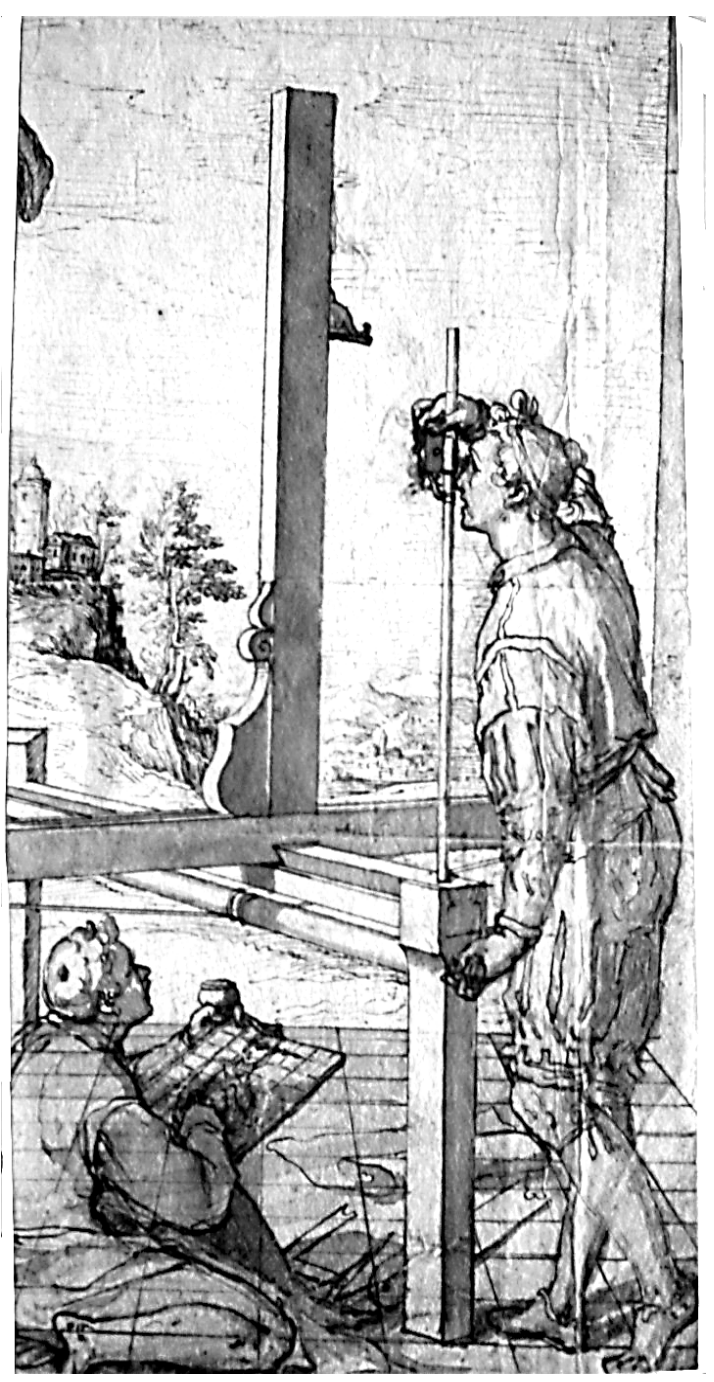

Fig. 4: Perspecteurs en plein air, dessin de l'entourage des Zuccaro, Varsovie, Muzeum Narodowe. 
tion de sa fonction. Cette scène figurée présente deux emplois du distanciomètre : le relevé topographique et le calcul des hauteurs à distance. Les opérateurs se trouvent dans un paysage collinaire, ponctué de forteresses et de bourgades. On pourrait s'interroger longtemps sur la singularité de cet objet qui se donne à voir à la fois comme opérationnel et figuratif.

Ce double statut fait planer un doute sur le caractère productif de l'objet. L'apparence luxueuse et soignée de cet exemplaire semble clairement désigner un emploi assez éloigné du terrain. Mais audelà, la polyvalence des fonctions laisse à penser que cet objet a pu servir à montrer la continuité de

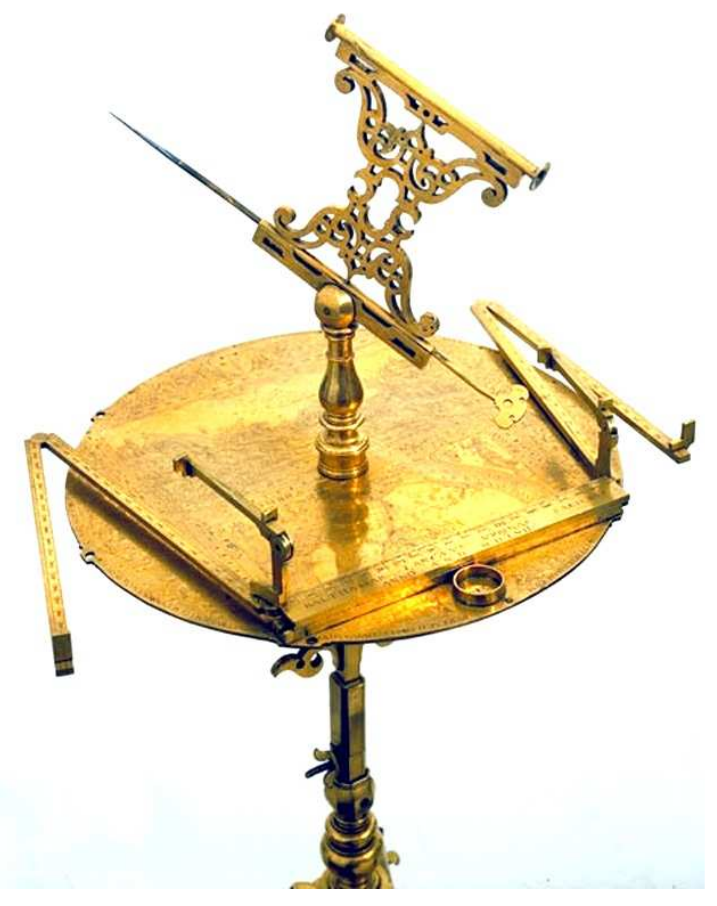

Fig. 5 : Le Distanciomètre de Baldassare Lanci, Florence, Museo Galileo. procédure entre le relevé triangulaire des distances et le relevé axonométrique des forteresses, d'où fut déduit par la suite le lien avec la perspective picturale. Cet instrument de représentation qui montre en image l'usage qu'il suggère sans nécessairement l'offrir aux praticiens, ouvre un débat sur les conditions d'exercice de ces machines de perspective.

La fameuse gravure des prospecteurs en plein air, dont Danti "a trouvé une esquisse dans les dessins de Vignole, mais sans aucun texte » 32 , nous conduit à poursuivre cette réflexion. L'instrument de grande taille, plus grand que la hauteur d'un homme, fonctionne sur le principe de celui inventé par Danti composé de réglettes en équerre. Cependant la composition de la scène, montrant des opérateurs en action, attire notre attention. Ils emploient l'instrument dans la nature pour dessiner une sculpture à l'antique juchée sur un piédestal. À l'arrière, se trouve un paysage représenté par plans et non en perspective : sur la droite, l'arbre à miparcours du tertre est deux fois plus grand que l'édifice situé à son sommet, pourtant à quelques dizaines de mètres seulement. Les bras qui composent l'instrument dans l'axe horizontal et dans l'axe vertical divisent en fait les trois plans de la scène. Le plan inférieur où se trouvent les deux opérateurs est un lieu intérieur, composé d'un dallage rappelant une iconographie de la perspective héritée d'Alberti. Sur la gauche, un paysage lointain, composé d'éléments minuscules, sans transition ni continuité avec l'espace des peintres, encadré par les bras de l'instrument. Sur la droite, l'espace des objets à représenter : le piédestal de la statue, posant encore sur le dallage, et à l'arrière le paysage composé du tertre avec son gigantesque arbre et son petit château. Regardons à présent la procédure de mise en œuvre de l'instrument: le peintre sur la gauche constate les coordonnées des points saillants de la sculpture observés à travers le viseur et les communique oralement au second opérateur qui les reporte sur une feuille mise au carreau.

Cette gravure illustre finement les paradoxes de la mécanisation de la perspective. Rationaliser et 
non reproduire le réel à travers un instrument fondé sur une certitude mathématique. Les formes de la sculpture-modèle, résultat d'un travail éprouvé par la «pratique » trouvent leur traduction rationnelle dans le dessin grâce à l'instrument perspectif, qu'il soit matériel ou intellectuel. On suggère ici que les paysages de l'arrière-plan, dans le chaos de la nature, sont également susceptibles de subir cette procédure qui, par l'art composé de règles et si possible de règles mathématiques, leur donnera leur cohérence.

Cette image nous porte à regarder à présent l'instrument le plus simple mais aussi le plus énigmatique de la série présentée par Danti, celui de Tommaso Laureti. Inséré dans la partie propédeutique du traité, avant les règles de Vignole et autres instructions pratiques que Danti leur a adjointes, cet instrument « montrera en pratique la vérité [...] du théorème sur la dégradation de la figure et [montrera] que l'œil voit le carré raccourci de la même manière que lorsqu'il est produit par les règles de Vignole »33.

Il est d'une facture technique très élémentaire, composé d'un cadre avec un bras central, placé perpendiculairement. Le cadre fait office de plan du tableau, l'extrémité du bras à angle droit, de point de l'œil. Depuis ce viseur, on observe le dallage à l'arrière du cadre, et l'on place dans le cadre des fils noirs entre les deux montants, correspondant aux

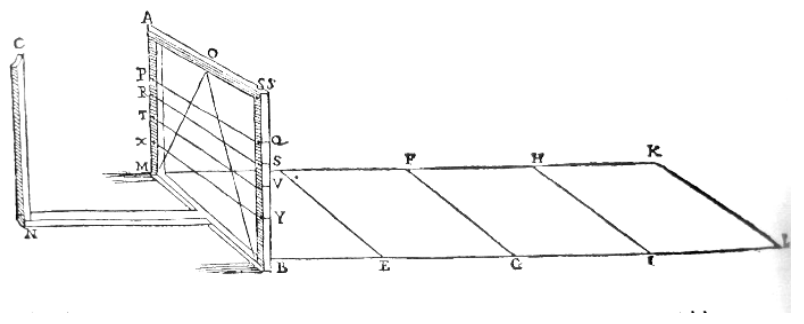

Fig . 6 : L'instrument de Tommaso Laureti, dans Le due regole della prospettiva pratica del Vignola, Rome, 1583, p. 39. partitions horizontales du dallage. Cet instrument n'est pas une machine, il n'est pas productif, il ne sert même pas à produire un dessin. Cet instrument a pour fonction de montrer la conformité entre l'expérience sensible et la théorie perspective qui en est issue. Cet appareil démontre donc, sans qu'il soit besoin de géométrie, la conformité de la règle de perspective avec l'expérience de la vision naturelle34.

Parmi les dispositifs techniques liés à l'affirmation de la discipline perspective, on ne trouve guère de "machines ", un terme réservé dans l'italien de la Renaissance à des organismes complexes, naturels ou artificiels, possédant une autonomie en soi. On rencontre en revanche une longue série " d'instruments », légitimés par l'exposé d'Alberti dans le De Pictura (1435). Leur développement technologique s'est nourri des échanges avec les instruments employés dans d'autres domaines, occupés eux aussi à la mesure de l'espace, à des échelles variables. Tous ces instruments cependant, exposés dans des traités imprimés, ne semblent pas avoir pour but de faciliter les opérations sur le chantier ou de se substituer à une pratique, c'est-à-dire à l'usage ou à une règle issue de la géométrie. Ces instruments, intermédiaires d'une opération dans l'acception la plus large, sont de nature intellectuelle ou matérielle. Les premiers nous renvoient aux règles. Les seconds sont apparentés à notre compréhension actuelle de la machine. Ces instruments mécaniques constituent un groupe encore large. Si la plupart d'entre eux n'a pas été conservée, on ne peut douter qu'ils aient été réalisés. Leur fonction pose cependant question. Certains sont clairement productifs alors que d'autres semblent assumer le rôle de démonstration et de validation par l'expérience d'une théorie rédigée. Le traité des instruments mécaniques de Levinus Hulsius, publié en fascicules à Francfort au début du XVII ${ }^{\mathrm{e}}$ siècle, devait également contenir un volume, le quatorzième, consacré à la perspective. Malheureusement, seuls les quatre premiers volumes virent le jour. Mais alors qu'il présentait les instruments des 
autres domaines sous l'aspect de l'usage que l'on pouvait en faire, il annonçait que "de nombreux différents auteurs qui ont écrit sur la perspective seraient cités et leurs instruments placés devant les yeux »35. Une galerie d'instruments en quelque sorte qui nous rappelle la tradition des théâtres de machines et ne semble encore rien préfigurer de la postérité que la mécanisation du dessin connaît par la suite, à travers les perspectographes qui, entre les XVII e ${ }^{e}$ XIX ${ }^{e}$ siècles, s'épanouissent sur de nouvelles bases, plus clairement opérationnelles.

${ }^{1}$ Vocabolario degli accademici della Crusca, Venise, Giovanni Alberti, 1612. Le lexique technique de la Renaissance a été peu étudié de façon systématique et les catégories linguistiques et classificatrices auxquelles les différentes machines correspondent encore moins. Les initiatives de l'École normale supérieure de Pise, à la fin des années soixante-dix, n'ont pas été poursuivies: Convegno Nazionale sui lessici tecnici delle arti e dei mestieri, Cortone, 1979; Convegno nazionale sui lessici tecnici del Sei e Settecento. Pise, 1980. Parmi les rares exceptions depuis dans le domaine de l'histoire de l'art, signalons, dans le sillage des travaux précédents : P. Barocchi, «Vasari e il lessico tecnico », Bollettino d'informazioni del Centro di Ricerche Informatiche per i Beni Culturali 1996, 6, p. 25-35.

${ }_{2}^{2}$ Les transcriptions systématiques des manuscrits de Léonard commencent à produire des résultats utilisables dans ce sens : $\mathrm{P}$. Manni, M. Biffi, Glossario leonardiano: nomenclatura delle macchine nei codici di Madrid e Atlantico, Florence, 2011. Consulter également le site internet www.leonardodigitale.com, à l'origine de cet ouvrage de consultation.

3 Milan, Biblioteca Trivulziana, Codex 2162, fol. 12v.

4 Paris, Institut de France, Codex B, fol. 33r.

5 «Oh speculatore di questra nostra macchina! Non ti contristare perché coll'altrui morte tu ne dia notizia, ma rallegrati che il nostro altore abbia fermo lo intelletto a tale eccellenzia di strumento ! » Windsor, Royal library, RL 19075v.

${ }^{6}$ Vocabolario degli accademici della Crusca, Florence, 1612, p. 496: "Il macchinare, che è ordinare, e apparecchiare, e dicesi sempre d'insidie, e simili cose / Per macchina, ordigno, e strumento da guerra / E macchina diciamo anche in signifi. d'edificio nobile, e grande ».

7 L. Ariosto, Orlando furioso, chant 11, Milan, 1976 [1516] vol. I, p. 228.
${ }^{8} \mathrm{G}$. Vasari, Le vite de' più eccellenti architetti, pittori, et scultori italiani..., Turin, 1991 [1550], vol. I, p. 213.

9 K. Gloy, Das Verständnis der Natur, Munich, 1995, I, p. 157$158,166$.

${ }^{10}$ Vocabolario..., 1612, p. 575 : « Cosa artificiosa, nome generico di strumento artificiosamente composto, per diverse operazioni ».

${ }^{11}$ A. Calepino, Il dittionario della lingua latina..., Venise, 1554, fol. 145v.; C. de Las Casas, Vocabulario de las dos lenguas toscana y castellana, Venise, 1576, p. 155.

${ }_{12}$ F. Alunno, Della fabbrica del mondo, Venise, 1562, fol. 3 r.

${ }_{13}$ Vocabolario..., 1612, p. 859 : « quello col quale o per mezzo del quale, noi operiamo »,

${ }^{14}$ Ibid, « Distinguimento determinato di quantità, e strumento, col qual si distingue », p. 534.

${ }^{15} \mathrm{Ibid}$, « Parte nobilissima dell'animale, strumento della vista », p. 565 .

${ }^{16}$ L. B. Alberti, La peinture, trad. Th. Golsenne et B. Prévost, Paris, 2004, p. 119.

${ }^{17}$ p. 121. On ne sait cependant pas si les réticences qu'il rapporte sont liées au caractère utilisable de l'intersecteur ou plus généralement à la théorie perspective.

${ }^{18}$ Ce dispositif est très proche de ceux à travers lesquels Léonard a présenté ses vues sur la vision, au moment où il développe un exposé général sur la perspective. Dans son discours sur la peinture, ces expédients de la pratique sont cependant loin d'être indispensables. Voir K. Veltman, Linear perspective and the visual dimensions of science and art, Munich, 1986, p. 107-112.

19 A. Dürer, Unterweisung der Messung, Munich, 1908, p. 187188 ; Instruction sur la manière de mesurer, trad. M. Van Peene, Paris, 1995, p. 196-198 ; Géométrie, trad. J. Peiffer, Paris, 1995, p. 249-352.

20 « La scala altimetra o la quadra zoppa, o qual si voglia altro instrumento, con cui si mira " La prospettiva di Euclide, E. Danti (trad.), Florence, 1573, p. 35. On trouve une revue de ces intruments employés à la Renaissance dans G. Vasari le Jeune, Raccolto ... di varii instrumenti per misurare con la vista, F. Camerota (ed.), Florence, 1996.

21 «I raggi visuali si riflettono ad angoli pari, tanto negli specchi piani, come anco ne' rotondi, e ne' concavi », ibid, p. 80.

22 Ibid, p. 81-84.

23 « Vedrai [...] le nugole dipinte caminare nella dipintura, come fanno in cielo, e le imagini delle persone, che passano per la piazza », ibid., p. 82. 
${ }^{24}$ S. Dupré, « Optics, Pictures and Evidence : Leonardo's Drawings of Mirrors and Machinery », Early Science and Medicine, 2, 2005, p. 211-236.

25 "Gli strumenti fatti dall'arte ci mostrono, che i raggi son portati per retta linea », ibid, p. 2.

${ }^{26}$ Les instruments présentés sont les suivants: le taglio de Tommaso Laureti, le portillon de Dürer, le portillon à Dioptre, le portillon à réglette de Girolamo Ruscelli, le double portillon d'Orazio Trigini dei Mari, les réglettes en équerre, le perspectographe du dessin circulaire de Zuccaro et le distanciomètre de Baldassare Lanci.

${ }^{27} \mathrm{E}$. Danti, Les deux règles de la perspective pratique de Vignole, trad. P. Dubourg Glatigny, Paris, 2003, p. 229.

${ }_{28}^{8}$ Ibid., p. 233.

${ }^{29}$ Florence, Museo Galileo, Inv. 152-3165.

зо F. Camerota, «Il distanziometro di Baldassarre Lanci : prospettiva e cartografia militare alla corte dei Medici », Musa musaei : studies on scientific instruments and collections in honour of Mara Miniati, Florence, 2003, p. 79-92.

${ }^{31}$ C. Maltese, « La prospettiva curva di Leonardo da Vinci e uno strumento di Baldassarre Lanci », in M. Dalai Emiliani (éd.), $L a$ prospettiva rinascimentale. Codificazioni e trasgressioni, Florence, 1980 , p. 417-425.

${ }^{22}$ Danti, Les deux règles...., p. 239.

33 Ibid., p. 195.

34 «Cet instrument montrera en pratique la vérité de ce que l'on a dit dans le théorème sur la dégradation de la figure et que l'œil voit le carré raccourci de la même manière » Ibid., p. 195.

35 « [Es] werden viel underschiedene Authores angezogen und ihre Instrumenta vor Augen gestellt so von der Perspectiff geschrieben haben », L. Hulsius, Tractat der mechanischen Instrumenten, Francfort, 1604, p. 6. 